\title{
Effect of pulsed electromagnetic field therapy on the osteogenic and adipogenic differentiation of bone marrow mesenchymal stem cells
}

\author{
T. Lu' ${ }^{1}$ Y.X. Huang ${ }^{2}$, C. Zhang', M.X. Chai ${ }^{1}$ and J. Zhang ${ }^{1}$ \\ ${ }^{1}$ Second Department of Orthopedic Surgery, \\ The First Affiliated Hospital of Xinxiang Medical University, Xinxiang, China \\ ${ }^{2}$ First Department of Orthopedic Surgery, \\ The First Affiliated Hospital of Xinxiang Medical University, Xinxiang, China \\ Corresponding author: T. Lu \\ E-mail: lutansdos@yeah.net \\ Genet. Mol. Res. 14 (3): 11535-11542 (2015) \\ Received January 13, 2015 \\ Accepted May 18, 2015 \\ Published September 28, 2015 \\ DOI http://dx.doi.org/10.4238/2015.September.28.5
}

\begin{abstract}
We investigated the effects of pulsed electromagnetic fields (PEMFs) of $20 \mathrm{~Hz} / 2 \mathrm{mT}$ on the osteogenic and adipogenic differentiation of bone marrow stem cells (BMSCs). Sprague Dawley rat BMSCs were isolated and cultured in vitro. The BMSCs of the third passage were obtained and stimulated by PEMFs of $20 \mathrm{~Hz} / 2 \mathrm{mT}$. The alkaline phosphatase (ALP) activity was measured according to the ALP assay kit manufacturer instructions, the BMSC osteogenic and adipogenic indicators were detected by semi-quantitative reverse transcription polymerase chain reaction (RT-PCR), and oil red O staining was used to observe the adipose-induced adipogenic differentiation of BMSCs. PEMFs of $20 \mathrm{~Hz} / 2 \mathrm{mT}$ significantly promoted the activity of ALP in the BMSCs $(\mathrm{P}<0.01)$ and mRNA expression of osteogenic proteins (osteocalcin and osteopontin). The PEMFs inhibited the expression of adipogenic transcription factors such as adipokines and
\end{abstract}


adipocyte-binding protein-2, and the adipogenic differentiation of BMSCs. PEMFs of $20 \mathrm{~Hz} / 2 \mathrm{mT}$ can promote osteogenic differentiation and inhibit adipogenic differentiation in BMSCs.

Key words: Pulsed electromagnetic field; Osteocalcin; Osteopontin; Alkaline phosphatase; Bone mesenchymal stem cells

\section{INTRODUCTION}

Pulsed electromagnetic fields (PEMFs) can enable current to flow through a Helmholtz coil to generate magnetic field effects with pulsed intervals that can simulate the microenvironment of physiological activities in organisms during motion (Marcheggiani Muccioli et al., 2013). The technique is safe, environmentally friendly, non-invasive, and simple; it also avoids the introduction of infection, has wide applicability, and has few complications. PEMFs have been used for nearly 40 years as a means of non-invasive treatment of fracture and bone nonunion. The osteogenic effect of the technique has been confirmed by in vivo clinical studies, but its mechanism of action has not yet been clarified (Assiotis et al., 2012; Teven et al., 2012). Bone marrow stem cells (BMSCs) are mesenchymal stem cells that exist in bone marrow (Yan et al., 2013). They are also a class of stem cells with the potential for directional or multi-directional differentiation, and can differentiate into bone cells, cartilage cells, or fat cells. These cells can differentiate into osteogenic tissue, adipose tissue, and many other tissues under the influence of different factors (Shi et al., 2011; Milanesi et al., 2012). We speculate that PEMFs can help promote the directional differentiation of BMSCs into osteogenic tissue and inhibit the differentiation of BMSCs into other tissues, thereby promoting the healing of fractures.

In this study, BMSCs were stimulated by PEMFs of $20 \mathrm{~Hz} / 2 \mathrm{mT}$ to explore the effectiveness of PEMFs at promoting osteogenic differentiation and inhibiting adipogenic differentiation in BMSCs in vitro.

\section{MATERIAL AND METHODS}

\section{Reagents and apparatus}

Dulbecco's modified Eagle's low glucose and high glucose media were purchased from Sigma (USA); high-quality fetal bovine serum was bought from the Kang Pu Huawei Technology (Beijing, China); trypsin was obtained from the Haoyuan Biotech (Shanghai, China); an alkaline phosphatase detection kit and a Coomassie brilliant blue protein assay kit were purchased from the Major Biotech (Shanghai, China); reverse transcriptase was bought from AMRESCO (USA), a polymerase chain reaction (PCR) kit was obtained from the Ruize Kang Biotech (Beijing, China); and Trizol was provided by the Shrek Biotech (Shanghai, China).

An electromagnetic field generator was purchased from GE (USA), an inverted phase contrast microscope was obtained from OLYMPUS (Tokyo, Japan), and the PCR instrument, the electrophoresis apparatus, the B10-RAD gel imaging analysis system, and the spectrophotometer were bought from Heraeus (Frankfurt, Germany). 


\section{Experimental animals}

Twenty 7-8-week-old Sprague Dawley rats of either gender with a body mass of 160$180 \mathrm{~g}$ were provided by the Experimental Animal Center of Beijing Medical University, Certificate of Conformity SCXK (Beijing, China) 2012-0047. The rats were randomly divided into a stimulation and a control group $(\mathrm{N}=10)$.

\section{Isolation and in vitro culture of BMSCs}

The rats were killed by cervical dislocation, soaked in $75 \%$ alcohol for $30 \mathrm{~min}$, and then the bilateral femurs were isolated on a sterile operating table. The muscle tissue surrounding the femur was removed, and both metaphyses, including the epiphyseal plates, were cut off. A 20-mL syringe was used to draw $10 \mathrm{~mL} 10 \%$ Dulbecco's modified Eagle's low glucose medium (containing $100 \mu \mathrm{g} / \mathrm{mL}$ penicillin and $100 \mu \mathrm{g} / \mathrm{mL}$ streptomycin) to wash the medullary cavity. After the bone marrow cell suspension had been pipetted repeatedly, the cells were counted. The cells were seeded in a $50-\mathrm{mL}$ culture flask directly after the cell density had been adjusted to approximately $2 \times 10^{6} \mathrm{cells} / \mathrm{mL}$, and were then incubated at $37^{\circ} \mathrm{C}$ in $5 \% \mathrm{CO}_{2}$ under saturated humidity. The medium was changed about $6 \mathrm{~h}$ after inoculation, and once every 2 days subsequently. Because the erythrocytes, leukocytes, and hematopoietic stem cells in the bone marrow washing fluid were mainly in suspension in vitro, they could be removed by monolayer cell culture and regular medium change. The remaining adherent cells were mainly BMSCs. The cells were digested with $0.5 \%$ trypsin for passage till they proliferated to a full monolayer 7 days later.

\section{Detection of alkaline phosphatase (ALP)}

After the BMSCs of the third passage had been obtained, they were seeded on six 6-well plates at $1 \times 10^{5}$ cells $/ \mathrm{mL}, 3 \mathrm{~mL}$ in each well, making a total of 30 wells ( 15 wells for the stimulation group and 15 for the control group). After the cells had grown to $80 \%$ confluence, the cells in the stimulation group were placed in the $20-\mathrm{Hz} / 2 \mathrm{mT}$ magnetic field for stimulation for $6 \mathrm{~h}$ each day. The cells from three wells were collected for both the stimulation and the control groups on days $2,4,6,8$, and 10 , washed with phosphate-buffered saline, digested, and centrifuged at 2000 revolutions per minute for $10 \mathrm{~min}$. After being centrifuged, the cells in each well were pipetted evenly with triple-distilled water, and then $0.5 \%$ TritonX-100 was used for lysis. The absorbance values at $595 \mathrm{~nm}$ were measured using a spectrophotometer according to the Coomassie brilliant blue protein assay kit manufacturer instructions to calculate the protein levels in the two groups. The absorbance values at $520 \mathrm{~nm}$ were then detected using the spectrophotometer according to the ALP detection kit manufacturer instructions, and finally the actual amount of ALP in each well was calculated through the detected protein levels based on the formula in the instructions, which was expressed as $\mathrm{U} / \mathrm{g}$ protein.

\section{Detection of effects of PEMFs by semi-quantitative reverse transcription-PCR (RT-PCR)}

The BMSCs of the third passage were seeded into eight culture flasks with cell densities of $1 \times 10^{5}$ cells $/ \mathrm{mL}$ (four flasks for the stimulation group and four for the control group). 
After the cells had grown to $80 \%$ confluence, the cells in the stimulation group were placed in the $20 \mathrm{~Hz} / 2 \mathrm{mT}$ magnetic field for stimulation for $6 \mathrm{~h}$ each day. A bottle of cells was collected from both the stimulation and control groups on days 3, 6, 9, and 12 for successive RNA extraction, RT, PCR, 5\% agarose gel electrophoresis, and gel imaging and analysis. The PCR primer sequences are shown in Table 1. The test described above was repeated twice.

\begin{tabular}{llcc}
\multicolumn{1}{l}{ Table 1. Polymerase chain reaction primer sequences. } \\
\hline Primer name & Primer sequence & Cycle & Product length (bp) \\
\hline Osteocalcin & 5'-TGGAATTCACGAAGGGTTTCGG-3' & 45 & 327 \\
Osteopontin & 5'-GGAGCCTCTCTAAGACCCCT-3' & 45 & 296 \\
Adipokine & 5'-CCCATATATGCTTTGCTA-3' & & 372 \\
AP-2 & 5'-TCTTCGAGTCGCTACCTC-3' & \\
& 5'-GCCGCAATAATCCTGAGTCCTCAA-3' & 45 & 406 \\
Glyceraldehyde phosphate dehydrogenase & 5'-CCCAAAGTGTTGGCCAACCTTTAA-3' & & 34 \\
& 5'-AATTCCGGGGCACTTAGGTCCAATCA-3' & 45 & 341 \\
\hline
\end{tabular}

AP-2 = adipocyte-binding protein-2.

\section{Observation of adipogenic induction by oil red $O$ staining}

The BMSCs of the third passage were seeded into 20 culture dishes of $35 \mathrm{~cm}^{2}$ at a cell density of $1 \times 10^{5}$ cells $/ \mathrm{mL}$ (10 dishes for the stimulation group and 10 for the control group). After the cells had grown to $80 \%$ confluence, the cells in both groups were added to adipose-induced medium, and the cells of the stimulation group were placed in the $20-\mathrm{Hz} / 2$ $\mathrm{mT}$ magnetic field for stimulation for $6 \mathrm{~h}$ each day. The cells were removed after 2 weeks and stained using oil red $\mathrm{O}$ (the cells were fixed in $10 \%$ neutral formalin for $10 \mathrm{~min}$, soaked in $60 \%$ isopropanol for $30 \mathrm{~min}$, the isopropanol was discarded, the cells were added to the oil red $\mathrm{O}$ for staining for $30 \mathrm{~min}$, washed with $60 \%$ isopropanol for $2 \mathrm{~min}$, washed with tap water for $3 \mathrm{~min}$, dried in air, and investigated using an inverted microscope) (Wang et al., 2011).

\section{Statistical analysis}

All data are reported as means \pm SD and were analyzed by SPSS 15.0. Univariate analysis of variance was used.

\section{RESULTS}

\section{Effects of PEMFs on ALP}

After stimulation by PEMFs, the ALP activity in the stimulation group was significantly higher than in the control group, and the peak appeared on about day 5 (Table 2).

Table 2. Alkaline phosphatase (ALP) activities in the two groups.

\begin{tabular}{|c|c|c|c|c|c|}
\hline \multirow[t]{2}{*}{ Group } & \multicolumn{5}{|c|}{ ALP activity } \\
\hline & 2 days & 4 days & 6 days & 8 days & 10 days \\
\hline $\begin{array}{l}\text { Control } \\
\text { Stimulation }\end{array}$ & $\begin{array}{l}13.56 \pm 1.57 \\
19.27 \pm 1.62^{*}\end{array}$ & $\begin{array}{l}14.12 \pm 1.38 \\
28.61 \pm 1.91 *\end{array}$ & $\begin{array}{l}13.24 \pm 1.50 \\
46.08 \pm 2.08^{*}\end{array}$ & $\begin{array}{l}13.78 \pm 1.49 \\
36.25 \pm 1.83^{*}\end{array}$ & $\begin{array}{l}14.05 \pm 1.40 \\
26.20 \pm 1.67 *\end{array}$ \\
\hline
\end{tabular}

Compared with the control group, ${ }^{*} \mathrm{P}<0.01$. 


\section{RT-PCR results}

After stimulation by PEMFs, the expression levels of osteocalcin and osteopontin mRNAs were higher in the stimulation group than in the control group. The expression level of osteocalcin mRNA was elevated on day 3, while that of osteopontin mRNA was increased on day 9. The adipogenic transcription of adipokine and adipocyte-binding protein-2 (AP-2) was inhibited by PEMFs stimulation. The results were consistent after repeated experiments. This indicates that PEMFs can promote osteogenic differentiation and suppress adipogenic differentiation in BMSCs (Figure 1).
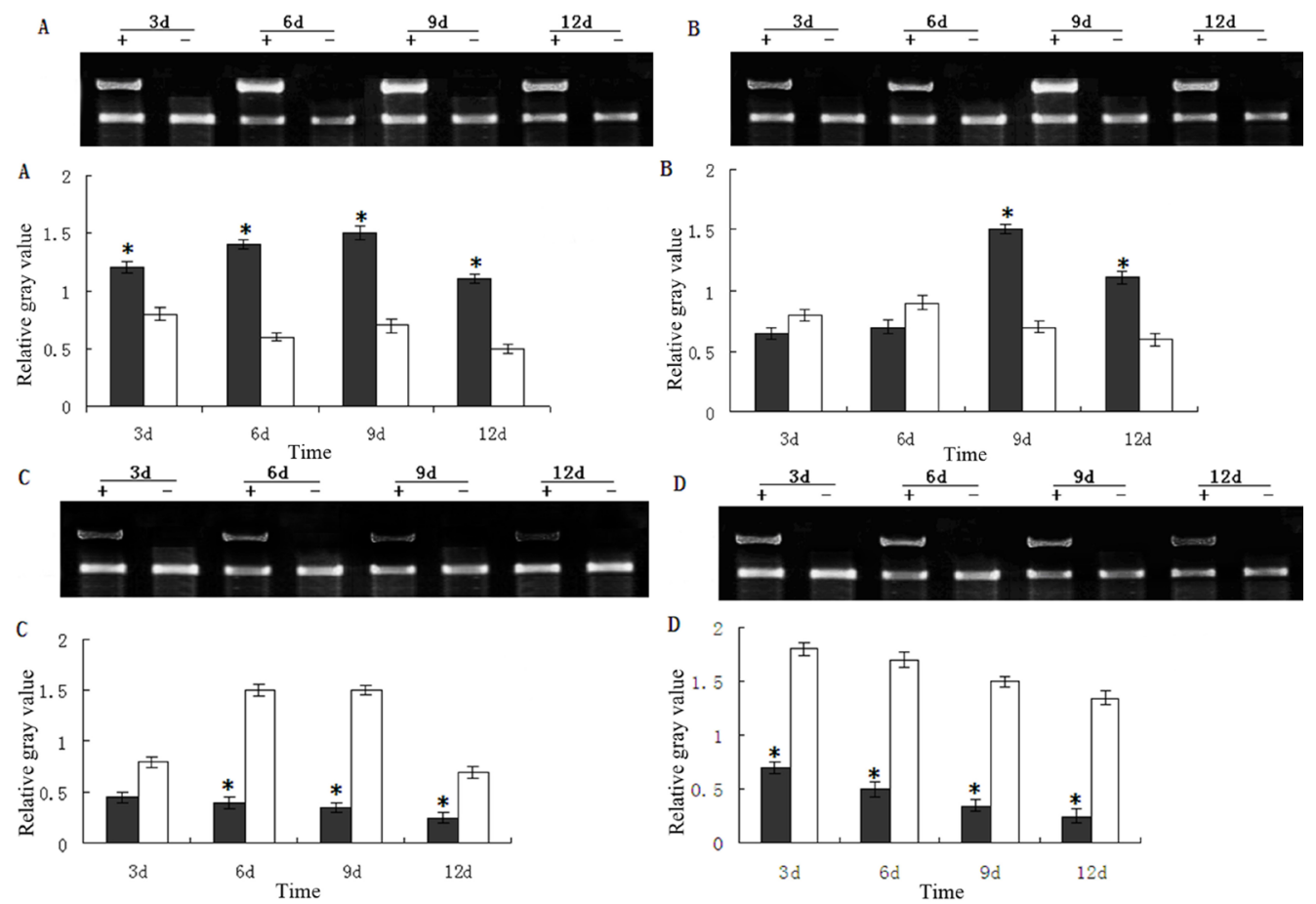

Figure 1. Expression levels of osteogenic and adipogenic indices stimulated by pulsed electromagnetic fields (PEMFs). A. Osteocalcin mRNA expression levels; B. osteopontin mRNA expression levels; C. adipocyte-binding protein-2 (AP-2) mRNA expression levels; D. adipokine mRNA expression levels. Compared with the control group, $* \mathrm{P}<0.05$.

\section{Oil red $O$ staining results}

Adipose-induced medium was added to further validate the effect of PEMFs in inhibiting the adipogenic differentiation of BMSCs. Two weeks later, a large number of lipid droplets could be seen in the BMSCs of the control group, and showed positive by oil red O staining; but there was no formation of lipid droplets in the stimulation group, and the cells were negative by oil red $\mathrm{O}$ staining, indicating that PEMFs can inhibit the adipogenic differentiation of BMSCs (Figure 2). 

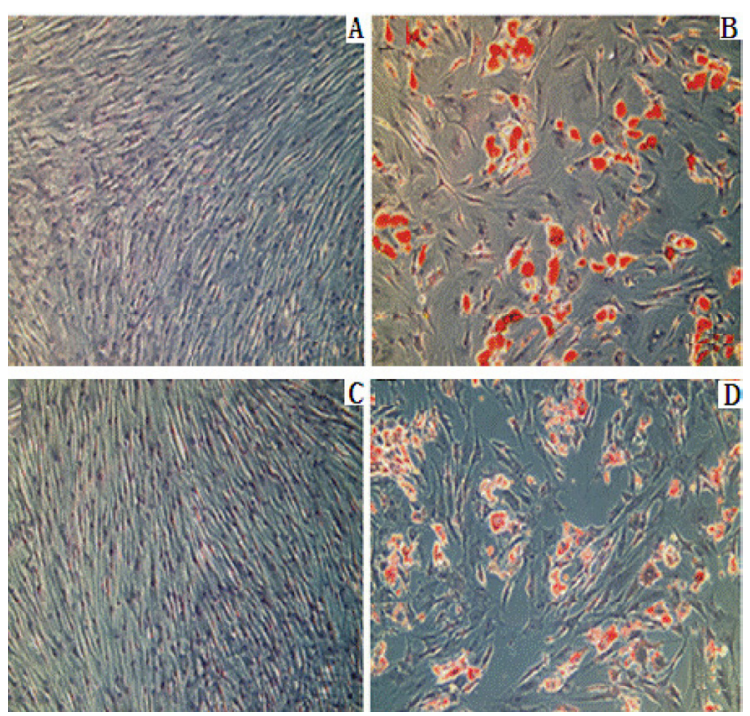

Figure 2. Oil red O staining results. A. Control group before staining (200X); B. control group after staining (200X); C. stimulation group before staining (200X); D. stimulation group after staining (200X).

\section{DISCUSSION}

Studies have shown that PEMFs can promote the expression of ALP and bone morphogenetic protein-2 (BMP-2) in BMSCs, and have the effect of promoting the differentiation of BMSCs into osteogenic tissue (Li et al., 2012). However, whether they can induce directional osteogenic differentiation of BMSCs has rarely been reported. To induce the differentiation of BMSCs into a certain kind of tissue cell, their differentiation into other types of cell must also be inhibited (Wakitani et al., 2011). ALP is the enzyme, and osteocalcin and osteopontin are the proteins that express the mature differentiation of osteoblasts, as well as signifying osteogenic differentiation in BMSCs (Fanburg-Smith et al., 2010; Okamura et al., 2013). Adipokine and AP-2 are two important transcription factors, and are often used as indicators of adipogenic differentiation of BMSCs (Zhu et al., 2012a,b). This study shows that PEMFs can promote the expression of a variety of osteogenic proteins in BMSCs, such as ALP, osteocalcin, and osteopontin. Moreover, PEMFs can also suppress the expression of adipokine, AP-2, and other adipogenic transcription factors in BMSCs. They can also suppress adipogenic differentiation in BMSCs under the influence of adipocyte medium. This suggests that PEMFs can promote osteogenic differentiation and inhibit adipogenic differentiation in BMSCs. In addition, it preliminarily indicates that PEMFs also have the effect of promoting the directional osteogenic differentiation of BMSCs.

A large number of clinical studies have shown that low-frequency PEMFs have an osteogenic effect, but high-frequency magnetic fields have a relatively weak influence on osteogenesis (Li et al., 2011; Ongaro et al., 2011). Studies have indicated that PEMFs of 15-30 $\mathrm{Hz}$ have the strongest osteogenic effect. Therefore, in this study we used PEMFs of $20 \mathrm{~Hz} / 2$ $\mathrm{mT}$ for BMSC stimulation (Sun et al., 2010). This study also shows that 20-Hz/2 mT PEMFs can promote the directional osteogenic differentiation of BMSCs.

Currently, there are many hypotheses about the osteogenic mechanism of PEMFs, 
such as the calcium ion effect and the endocrine theory. The endocrine theory postulates that PEMFs can promote cells' release of transforming growth factor- $\beta$, prostaglandin E2, etc. (Hannemann et al., 2011). To the best of our knowledge, this study reports for the first time that PEMFs can inhibit the adipogenic differentiation of BMSCs. Even if the cells are induced by adipocyte medium, the mechanism may still be related to the release of cytokines. Esposito et al. (2012) used PEMFs to stimulate BMSCs and found that the level of intracellular adenosine cyclophosphate was significantly elevated, and the expression level of BMP-2 was also increased. Some studies have found that prostaglandin E can inhibit chondrocyte differentiation and maturation by adenosine cyclophosphate-dependent protein kinase A and protein kinase C pathways (Ding et al., 2012). Chalidis et al. (2011) also found that the effect of BMP-2 on osteogenesis occurs through the activation of the adenosine cyclophosphate-dependent protein kinase A pathway. This suggests that adenosine cyclophosphate and its dependent protein kinase A pathway may play a vital role in the osteogenic effect of PEMFs, which may also be the basic pathway by which PEMFs suppress adipogenic differentiation of BMSCs. This was the focus of our research in the next step.

Compared with drugs and transcription factors, the osteogenic effect of PEMFs is superior in its non-invasiveness and ease of regulation in vitro (van der Jagt et al., 2012). The use of PEMFs to stimulate the support vector attached with stem cells to induce their directional osteogenic differentiation and to transfer them to the bone defect so as to promote the healing of factures is a new approach. The technique is also easy to control in vitro and provides a good means of bone tissue engineering. This study only explored the effects of PEMFs on promoting osteogenic differentiation and inhibiting adipogenic differentiation in BMSCs. However, whether PEMFs can suppress the differentiation of BMSCs into other kinds of cell (e.g., muscle cells and neuroblasts) requires further investigation.

\section{Conflicts of interest}

The authors declare no conflict of interest.

\section{REFERENCES}

Assiotis A, Sachinis NP and Chalidis BE (2012). Pulsed electromagnetic fields for the treatment of tibial delayed unions and nonunions. A prospective clinical study and review of the literature. J. Orthop. Surg. Res. 7: 24.

Chalidis B, Sachinis N, Assiotis A and Maccauro G (2011). Stimulation of bone formation and fracture healing with pulsed electromagnetic fields: biologic responses and clinical implications. Int. J. Immunopathol. Pharmacol. 24: 17-20.

Ding S, Peng H, Fang HS, Zhou JL, et al. (2012). Pulsed electromagnetic fields stimulation prevents steroid-induced osteonecrosis in rats. BMC Musculoskelet. Disord.12: 215.

Esposito M, Lucariello A, Riccio I and Riccio V (2012). Differentiation of human osteoprogenitor cells increases after treatment with pulsed electromagnetic fields. In Vivo 26: 299-304.

Fanburg-Smith JC, Auerbach A, Marwaha JS, Wang Z, et al. (2010). Reappraisal of mesenchymal chondrosarcoma: novel morphologic observations of the hyaline cartilage and endochondral ossification and beta-catenin, Sox 9 , and osteocalcin immunostaining of 22 cases. Hum. Pathol. 41: 653-662.

Hannemann P, Göttgens KW, van Wely BJ, Kolkman KA, et al. (2011). Pulsed Electromagnetic Fields in the treatment of fresh scaphoid fractures. A multicenter, prospective, double blind, placebo controlled, randomized trial. $B M C$ Musculoskelet. Disord. 12: 90.

Li S, Luo Q, Huang L, Hu Y, et al. (2011). Effects of pulsed electromagnetic fields on cartilage apoptosis signalling pathways in ovariectomised rats. Int. Orthop. 35: 1875-1882.

Li X, Zhang M, Bai L, Bai W, et al. (2012). Effects of $50 \mathrm{~Hz}$ pulsed electromagnetic fields on the growth and cell cycle arrest of mesenchymal stem cells: an in vitro study. Electromagn. Biol. Med. 31: 356-364. 
Marcheggiani Muccioli GM, Grassi A, Setti S, Filardo G, et al. (2013). Conservative treatment of spontaneous osteonecrosis of the knee in the early stage: pulsed electromagnetic fields therapy. Eur. J. Radiol. 82: 530-537.

Milanesi A, Lee JW, Li Z, Da Sacco S, et al. (2012). $\beta$-Cell regeneration mediated by human bone marrow mesenchymal stem cells. PLoS One 7: e42177.

Okamura H, Yoshida K, Yang D and Haneji T (2013). Protein phosphatase 2A C $\alpha$ regulates osteoblast differentiation and the expressions of bone sialoprotein and osteocalcin via osterix transcription factor. J. Cell Physiol. 228: 1031-1037.

Ongaro A, Pellati A, Masieri FF, Caruso A, et al. (2011). Chondroprotective effects of pulsed electromagnetic fields on human cartilage explants. Bioelectromagnetics. 32: 543-551.

Shi SY, Ying XZ, Hu DX, Zheng Q, et al. (2011). [Experiment on articular cartilage defect repaired with autologous cancellous bone or cancellous bone enriching bone marrow stem cell]. Zhongguo Gu Shang. 24: 332-335.

Sun LY, Hsieh DK, Lin PC, Chiu HT, et al. (2010). Pulsed electromagnetic fields accelerate proliferation and osteogenic gene expression in human bone marrow mesenchymal stem cells during osteogenic differentiation. Bioelectromagnetics 31: 209-219.

Teven CM, Greives M, Natale RB, Su Y, et al. (2012). Differentiation of osteoprogenitor cells is induced by highfrequency pulsed electromagnetic fields. J. Craniofac. Surg. 23: 586-593.

van der Jagt OP, van der Linden JC, Waarsing JH, Verhaar JA, et al. (2012). Systemic treatment with pulsed electromagnetic fields do not affect bone microarchitecture in osteoporotic rats. Int. Orthop. 36: 1501-1506.

Wakitani S, Okabe T, Horibe S, Mitsuoka T, et al. (2011). Safety of autologous bone marrow-derived mesenchymal stem cell transplantation for cartilage repair in 41 patients with 45 joints followed for up to 11 years and 5 months. $J$. Tissue Eng. Regen. Med. 5: 146-150.

Wang Y, Goulart RA and Pantanowitz L (2011). Oil red O staining in cytopathology. Diagn. Cytopathol. 39: 272-273.

Yan Z, Zeng L, Li Z, Zhang H, et al. (2013). Bone marrow-derived endothelial progenitor cells promote hematopoietic reconstitution after hematopoietic stem cell transplantation. Transplant. Proc. 45: 427-433.

Zhu X, Du J and Liu G (2012a). The comparison of multilineage differentiation of bone marrow and adipose-derived mesenchymal stem cells. Clin. Lab. 58: 897-903.

Zhu X, Shi W, Tai W and Liu F (2012b). The comparition of biological characteristics and multilineage differentiation of bone marrow and adipose derived Mesenchymal stem cells. Cell Tissue Res. 350: 277-287. 